\title{
Simultaneous multiple-calf allonursing by a wild Masai giraffe
}

\author{
Bond, Monica L ; Lee, Derek E
}

\begin{abstract}
INTRODUCTION Allonursing is when mothers nurse young that are not their own. It is rarely seen in wild giraffes (Giraffa camelopardalis). Pratt and Anderson (1979) reported that of 860 observations of nursing attempts, 37 were by an unrelated calf, and just one succeeded in sustained nursing. Saito and Idani (2018) documented only five of 76 allonursing attempts resulted in sustained nursing. Typically, the mother appeared unaware that the nursing calf was not her own, and thus, these studies concluded that the instigators of allonursing were unrelated calves stealing milk from unwitting mothers. Here, we report an instance of simultaneous allonursing by three giraffe calves in Tarangire National Park, Tanzania. This case is unusual in that the lactating female appeared to be aware of all three nursing calves and allowed nursing for an extended period of time ( $>1 \mathrm{~min})$. 2 METHODS Since 2011, we systematically collected data on giraffes during 6 road-transect surveys per year in a 1,500-km2 area of the Tarangire Ecosystem, northern Tanzania, between latitude $3.27-4.08^{\circ} \mathrm{S}$ and longitude $35.73-$ $36.23^{\circ} \mathrm{E}$. The main vegetation communities are Acacia tortilis parkland, Acacia-Commiphora woodland, gall Acacia drepanolobium woodland, Combretum-Dalbergia woodland and open grassland (Lamprey, 1963). We identified individual giraffes from photographs using their unique and unchanging coat patterns (Foster, 1966) with the aid of pattern recognition software (Bolger, Morrison, Vance, Lee, Farid, 2012). We did not genetically assign calves to mothers, but we assumed mother-calf pairs from extended suckling behaviour $(>5 \mathrm{~s})$ without rejection. 3 RESULTS AND DISCUSSION We documented 82 extended nursing bouts during 45 surveys from 2011 to 2019. All previous bouts were a single female and single calf. On 27 January 2019, we witnessed a remarkable case of simultaneous multiple-calf allonursing. An adult female moved towards a group of calves ( $<1$ year of age), and three of the calves immediately ran over and began suckling from her. We were not certain whether any of the calves were her offspring nor whether the calves were related to each other or to the female. The calves were slightly different sizes, and thus, we presumed different ages, and two were females, and one was a male (Figure 1). The adult female allowed this nursing for over a minute and did not actively reject the nursing calves. She appeared to be aware of the situation as all three calves approached her from the front and were in her view during nursing (Figure 1).
\end{abstract}

DOI: https://doi.org/10.1111/aje.12673

Posted at the Zurich Open Repository and Archive, University of Zurich

ZORA URL: https://doi.org/10.5167/uzh-171319

Journal Article

Accepted Version

Originally published at:

Bond, Monica L; Lee, Derek E (2020). Simultaneous multiple-calf allonursing by a wild Masai giraffe. African Journal of Ecology, 58(1):126-128. 
DOI: https://doi.org/10.1111/aje.12673 


\section{Simultaneous multiple-calf allonursing by a wild Masai giraffe}

\section{RH: Multi-calf allonursing in giraffes}

4 Monica L. Bond ${ }^{1,2}$ and Derek E. Lee ${ }^{1,3}$

$5 \quad{ }^{1}$ Wild Nature Institute, Concord, NH, U.S.A.

$6{ }^{2}$ Department of Evolutionary Biology and Environmental Studies, University of Zürich, Zürich,

$7 \quad$ Switzerland

$8 \quad{ }^{3}$ Department of Biology, Pennsylvania State University, University Park, PA, U.S.A.

$9 \quad$ Key words: allonursing, giraffe, Giraffa camelopardalis

\section{Introduction}

11 Allonursing is when mothers nurse young that are not their own. It is rarely seen in wild giraffes

12 (Giraffa camelopardalis). Pratt and Anderson (1979) reported that of 860 observations of

13 nursing attempts, 37 were by an unrelated calf, and just one succeeded in sustained nursing. Saito 14 and Idani (2018) documented only five of 76 allonursing attempts resulted in sustained nursing. 15 Typically the mother appeared unaware that the nursing calf was not her own, thus these studies 16 concluded that the instigators of allonursing were unrelated calves stealing milk from unwitting 17 mothers.

18 Here we report an instance of simultaneous allonursing by three giraffe calves in Tarangire

19 National Park, Tanzania. This case is unusual in that the lactating female appeared to be aware of 20 all three nursing calves and allowed nursing for an extended period of time (> $1 \mathrm{~min})$. 


\section{Methods}

22 Since 2011, we systematically collected data on giraffes during 6 road-transect surveys per year 23 in a 1,500-km² area of the Tarangire Ecosystem, northern Tanzania, between latitude $3.27^{\circ} \mathrm{S}$ to

$244.08^{\circ} \mathrm{S}$ and longitude $35.73{ }^{\circ} \mathrm{E}$ to $36.23^{\circ} \mathrm{E}$. The main vegetation communities are Acacia tortilis 25 parkland, Acacia-Commiphora woodland, gall Acacia drepanolobium woodland, Combretum26 Dalbergia woodland, and open grassland (Lamprey, 1963). We identified individual giraffes

27 from photographs using their unique and unchanging coat patterns (Foster, 1966) with the aid of 28 pattern-recognition software (Bolger et al., 2012). We did not genetically assign calves to 29 mothers, but we assumed mother-calf pairs from extended suckling behavior $(>5 \mathrm{~s})$ without 30 rejection.

\section{$31 \quad$ Results and discussion}

32 We documented 82 extended nursing bouts during 45 surveys from 2011-2019. All previous

33 bouts were a single female and single calf. On 27 January 2019, we witnessed a remarkable case 34 of simultaneous multiple-calf allonursing. An adult female moved towards a group of calves $(<1$ 35 year of age), and three of the calves immediately ran over and began suckling from her. We were 36 not certain whether any of the calves were her offspring, nor whether the calves were related to 37 each other or to the female. The calves were slightly different sizes, thus we presumed different 38 ages, and two were females and one was a male (Fig. 1). The adult female allowed this nursing 39 for over a minute and did not actively reject the nursing calves. She appeared to be aware of the 40 situation as all three calves approached her from the front and were in her view during nursing 41 (Fig. 1). 
42 Allonursing in captive giraffes was reported, both in a published study (Gloneková et al., 2016)

43 and in anecdotal reports provided to us. In contrast, sustained allonursing was uncommon in wild

44 giraffes (Langmann, 1977; Pratt \& Anderson, 1979; Gloneková et al., 2017; Saito \& Idani,

45 2018). In wild giraffes, non-filial calves who initiated allonursing attempts joined only after the

46 filial calf began to suckle, and were never successful when approaching a female by themselves

47 (Saito \& Idani, 2018). Such allonursings appeared to be milk theft. This was the first instance

48 where we documented more than one calf allowed to suckle, and is consistent with the rarity of

49 allonursing from previous studies. To our knowledge this is the first record of three calves

50 nursing simultaneously from a single wild adult giraffe, and we believe the female was aware of

51 all three calves. Given that the calves rushed to her, we suspect she has nursed these calves

52 previously.

53 Giraffes typically give birth to a single calf (Dagg, 1971); twins are extremely rare and although

54 one usually dies it is possible that two of the allonursing calves were filials. We therefore

55 entertain three calf scenarios: (1) all non-filial calves; (2) one filial and two non-filials; and (3) 2

56 filials and one non-filial. From these scenarios we propose 6 potential explanations for the

57 female's allonursing behaviour. The adult female may be evacuating milk from her udder (1) that

58 her own calf did not drink or (2) she may have lost her calf and still has milk that she is sharing

59 with calves in her herd (Roulin, 2002). (3) The two smaller calves may be twins and the larger

60 third calf is stealing milk, as it is standing in the position typical for milk theft, further from the

61 female's view (Saito \& Idani, 2018). She may be allowing related or unrelated calves to

62 allonurse: (4) to improve survival of calves related to her (Hamilton, 1964) or (5) for reciprocity

63 (Trivers, 1971). (6) Allonursing can further be non-adaptive, as a by-product of providing care in

64 a group-living context (Jamieson, 1989; Packer et al., 1992; Packer \& Pusey, 1994). 
Total reproductive investment in filial offspring for monotocous species like giraffes is high (MacLeod \& Lukas, 2014), and lactation is more energetically costly than other types of parental care (König, 1997). Giraffe calves grow quickly to escape predation (Foster \& Dagg, 1971). Allonursing is therefore costly if the female's own calf is deprived of milk it needs for rapid growth (Roulin, 2002), which may explain why this behavior is so rare in the wild. However, giraffe calves form crèches accompanied by one or a few older females (Dagg \& Foster, 1976), indicating some level of cooperative care in this species, thus females may occasionally allow allonursing if benefits outweigh costs. Female giraffes tend to associate with related females (Bercovitch \& Berry, 2012; Carter et al., 2013), so allonursing may impart inclusive fitness benefits if the non-filial calves share her genes (Hamilton, 1964). Benefits may also include reciprocity, whether or not the allosucklers are related to her, if her behavior is reciprocated towards her current (or future) calf by other females at some later date (Trivers, 1971, but see Gloneková et al., 2016 which found no evidence of quantitative reciprocity in a captive giraffe population with high allonursing rates). Ungulates can discriminate kin (Cassinello \& Calabuig, 2008), and given that they benefit from living in social groups with their calves reared in crèches, allonursing might be a rare but inevitable consequence of group rearing. The costs of always rejecting non-filial calves might be higher that the costs of allowing some milk theft.

\section{Acknowledgements}

We thank B. König, F. Bercovitch, and 2 anonymous reviewers for helpful comments on the manuscript. We carried out the field research with permission from the Tanzania Commission for Science and Technology (COSTECH), Tanzania National Parks (TANAPA), and the Tanzania Wildlife Research Institute (TAWIRI). Research funding was provided by University of Zürich, Pennsylvania State University, Sacramento Zoo, Columbus Zoo, Tulsa Zoo, Tierpark Berlin and 
Zoo Berlin, African Wildlife Foundation, Cincinnati Zoo, Parrotia, Temperatio, Promotor Foundation, and Claraz Foundation.

\section{References}

Bercovitch, F.B., \& Berry, P.S.M. (2012) Herd composition, kinship and fission-fusion social dynamics among wild giraffe. African Journal of Ecology 51, 206-216.

Bolger, D.T., Morrison, T.A., Vance, B., Lee, D.E., \& Farid, H. (2012) A computer-assisted system for photographic mark-recapture analysis. Methods in Ecology and Evolution 3, 813822.

Carter, K.D., Seddon, J.M., Frère, C.H., Carter, J.K., \& Goldizen, A.W. (2013) Fission-fusion dynamics in wild giraffes may be driven by kinship, spatial overlap and individual social preferences. Animal Behaviour 85, 385-394.

Cassinello, J., \& Calabuig, G. (2007) Spatial association in a highly inbred ungulate population: evidence of fine-scale kin recognition. Ethology 114, 124-132.

Dagg, A.I. (1971) Mammalian Species, Giraffa camelopardalis 5, 1-8.

Dagg, A.I., \& Foster, J.B. (1976) The Giraffe: Its Biology, Behavior, and Ecology. Van Nostrand Reinhold, New York.

Foster, J.B. (1966) The giraffe of Nairobi National Park: Home range, sex ratios, the herd, and food. African Journal of Ecology 4, 139-148.

Foster, J.B., \& Dagg, A.I. (1971) Notes on the biology of the giraffe. East African Wildlife Journal 10, 1-16.

Gloneková, M., Brandlová, K., \& Pluháček, J. (2016) Stealing milk by young and reciprocal mothers: high incidence of allonursing in giraffes, Giraffa camelopardalis. Animal Behaviour $113,113-123$. 
111 Gloneková, M., Vymyslická, P.J., Žáčková, M., \& Brandlová, K. (2017) Giraffe nursing

112 behaviour reflects environmental conditions. Behaviour 154, 115-129.

113 Hamilton, W.D. (1964) The genetical evolution of social behaviour (I and II). Journal of

$114 \quad$ Theoretical Biology 7, 1-52.

115 Jamieson, I.G. (1989) Behavioral heterochrony and the evolution of birds' helping at the nest: an 116 unselected consequence of communal breeding? American Naturalist 133, 394-406.

117 König, B. (1997) Cooperative care of young in mammals. Naturwissenschaften 84, 95-104.

118 Lamprey, H.F. (1963) The Tarangire Game Reserve. Tanganyika Notes and Records 60, 10-22.

119 Langman, V.A. (1977) Cow-calf relationships in giraffe (Giraffa camelopardalis). Zeitschrift für 120 Tierpsychologie 43, 264-286.

121 MacLeod, K.J., \& Lukas, D. (2014) Revisiting non-offspring nursing: allonursing evolves when 122 the costs are low. Biology Letters 10, 20140378.

123 Packer, C., \& Pusey, A. (1994) Non-offspring nursing in social carnivores: minimizing the costs. $124 \quad$ Behavioral Ecology 5, 362-374.

125 Packer, C., Lewis, S., \& Pusey, A. (1992) A comparative analysis of non-offspring nursing. 126 Animal Behaviour 43, 265-281.

127 Pratt, D.M. \& Anderson, V.H. (1979) Giraffe cow-calf relationships and social development of 128 the calf in the Serengeti. Ethology 51, 233-251.

129 Roulin, A. (2002) Why do lactating females nurse alien offspring? A review of hypotheses and 130 empirical evidence. Animal Behaviour, 63, 201-208.

131 Saito, M. \& Idani, G. (2018) Suckling and allosuckling behavior in wild giraffe (Giraffa 132 camelopardalis tippelskirchi). Mammalian Biology 93, 1-4. 
133 Trivers, R.L. (1971) The evolution of reciprocal altruism. Quarterly Review of Biology 46, 35-

134 57. 


\section{Figure captions}

137 Fig. 1. Three calves simultaneously nursing from an adult female Masai giraffe (Giraffa

138 camelopardalis tippelskirchi) in Tarangire National Park, Tanzania on 27 January 2019.

139 Photo credit: Monica L. Bond.

140 


\section{Figure 1}

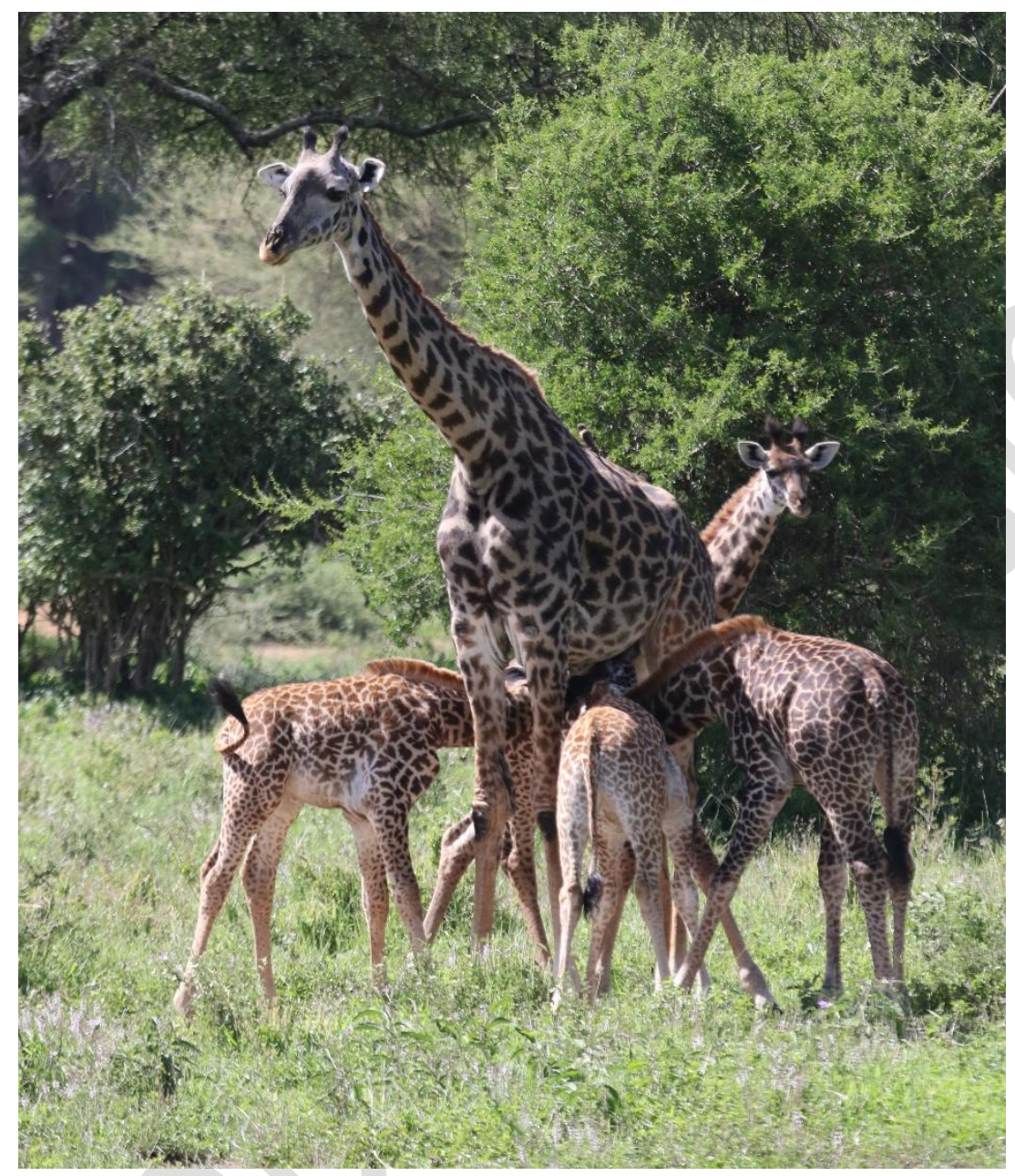

